\title{
PROSPETTIVA CRISTOCENTRICA DELLA CREAZIONE NEL NUOVO TESTAMENTO
}

A somiglianza degli scritti veterotestamentari, anche quelli neotestamentari presuppongono la fede nell'azione creatrice di Dio, senza propriamente dimostrarne in maniera argomentativa la validità. L'intreccio tra creazione e storia di salvezza è indispensabile per comprendere il messaggio sulla creazione sia nell'Antico Testamento, come nel Nuovo, che infatti è incentrato sull'annuncio di salvezza compiuta nella persona e storia di Gesù di Nazareth, Cristo e Signore mediante la risurrezione.

Nei suoi enunciati relativi alla teologia della creazione il Nuovo Testamento ricollega la fede della comunità cristiana con la fede dei padri e dà di questa una propria interpretazione incentrata sull'evento di Cristo. Gli scritti neotestamentari annunciano il "Dio che dà vita ai morti e chiama all'esistenza le cose che ancora non esistono" ( $\mathrm{Rm} 4,17)$. Il centro del messaggio neotestamentario, l'annuncio delle azioni salvifiche di Dio in favore degli uomini mediante la sua azione salvifica verso Gesù Cristo e in Gesù Cristo, in passi importanti del Nuovo Testamento è basato sulla teologia della creazione e da questa mediato. L'interesse verte tutto su Gesù Cristo e sulla portata salvifica della sua persona e delle sua azione.

L'evento di Cristo viene presentato dal Nuovo Testamento come evento escatologico, culmine insuperabile, di diritto e di fatto, della rivelazione di Dio e di tutto il dinamismo salvifico che parte dalla creazione. Sarebbe pertanto vano cercare nel Nuovo Testamento una riflessione sulla creazione che non tenesse conto dell'evento decisivo di Cristo. Come nell'Antico Testamento l'emergere della fede nella creazione avviene a partire dall'esperienza storico-salvifica, così nel Nuovo Testamento la comprensione storico-salvifica di Cristo precede e prepara la comprensione cosmico-creatrice. Osserviamo quindi un medesimo dinamismo teologico, sia nell'Antico come nel Nuovo Testamento. La riflessione sulla creazione deriva dalla riflessione sulla salvezza. La differenza specifica sta nel fatto che la salvezza è indissolubilmente legata all'evento di Cristo'.

M. Delmirani, Creazione in Cristo. Aspetti fondamentali di Teologia biblica sulla Creazione, Roma 1986. I testi del Nuovo Testamento riguardo "Creazione in Cristo" che vogliamo analizzare son ben noti: Gv 1,1-18, 1 Cor 8,6; Col 1,15-20; Eb 1,1-3. Pur appartenendo al corpus paolino e al vangelo di Giovanni, in realtà si tratta dei passi eleborati dalla comunità cristiana primitiva sotto forma di confessioni di fede (1Cor e Eb) e di inni cristologici ( $\mathrm{Col}$ e $\mathrm{Gv})$. Ci rendiamo così conto di trovarci di fronte a formule solenni, titoli cristologici ed espressioni poetiche. Il tutto risulta molto evocativo, ma nello stesso tempo vago e privo di precisione. Sarebbe dunque vano cercarvi rigore teologico. Si dovranno al contrario leggere per quello che sono, cioè come testi di commossa esalta- 


\section{La cristologia del prologo giovanneo (Gv 1,1-18)}

Prendiamo all'inizio in considerazione soprattutto alcuni aspetti del prologo giovanneo: Gv 1, 1-18, per l'importanza singolare che questo testo riveste nella dottrina cristiana della creazione ${ }^{2}$. Gli elementi che ci fanno leggere il prologo in chiave di una teologia della creazione sono diversi. In primo luogo, è da notare il puntuale

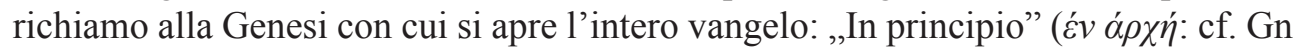
1,1). Al versetto 3 si trova un esplicito richiamo alla creazione mediante la parola; ai versetti $4 \mathrm{~s}$ il tema della luce e delle tenebre. L'intero primo capitolo è costruito sulla cadenza di una settimana che descriverebbe la „nuova creazione” operata da Gesù, culminante nella manifestazione della gloria di Gesù e nella fede dei discepoli, che è il principio vitale dell'uomo nuovo $(2,11)$.

Si deve notare, però, che il „principio” di Gv 1,1 è diverso rispetto a Gn 1,1. In Genesi infatti si parlava del principio temporale della creazione, mentre Giovanni allude a un'origine eterna del Logos Dio. Si parte quindi dalla sua preesistenza (come in Col 1,15-20 e Eb 1,1-3), e in base ad essa si parla della creazione, con precisa allusione ai testi sulla sapienza preesistente ( $\operatorname{Pr} 8,22-31$; Sir 2-1,3-12; Sap 9,9-12.18). Si mette in evidenza così un itinerario del Logos, che parte da Dio e a lui ritorna (versetti 1-2.18), per comunicare all'uomo il dono di Dio (versetti $12 \mathrm{~s}$. $)^{3}$. Giovanni mette in rilievo l'unità fra la parola creatrice di $\mathrm{Gn} 1,1$ e la parola incarnata, fondendo nel concetto di Logos elementi provenienti da ambiti diversi. Il dabar biblico, le speculazioni giudaiche sulla sapienza, forse anche le tematiche gnostiche e le idee stoiche sul Logos. L'originalità consiste nel fatto che questo Logos è presentato dal IV vangelo con caratteristiche personali, incarnato in Gesù di Nazaret e dotato delle stesse caratteristiche divine di Dio, presso il quale esiste dall'eternità.

Il ruolo creatore del Logos è affermato al v. 3: „Tutto è stato fatto per mezzo di lui, e senza di lui niente è stato fatto di tutto ciò che esiste". Tutto è stato creato „per mezzo” ( $\delta l \alpha ́)$ del Logos e, d'altro lato, è per mezzo ( $\delta i \alpha ́)$ di Gesù Cristo che sono state date all'uomo „la grazia e la verità” (v. 17). Il medesimo Logos creatore è anche il salvatore, passando però attraverso l'incarnazione (v. 14). Inoltre, come il

zione di Gesù Cristo. Pertanto, anche il loro ambiente vitale (Sitz im Leben) che si ritiene con tutta la probabilità di carattere liturgico conduce nella stessa direzione di lettura. $\mathrm{Cf}$. G. Barbaglio, Creazione, in: Nuovo Dizionario di Teologia, a cura di G. Barbaglio e S. Dianich, Roma 19947, p. 209; Cz. Bartnik, Dogmatyka katolicka, vol. 1, Lublin 1999, p. 284ss.

2 Per una presentazione della dottrina giovannea si confronti: J. Mateos - J. Barreto, Dizionario teologico del Vangelo di Giovanni, Assisi 1982, p. 56-65; R. Schnackenburg, Il vangelo di Giovanni, Brescia 1973; I. De La Potterie, Studi di cristologia giovannea, Genova 1986; G. Neyrand, Le sens des "logos" dans le prologue de Jean, "Nouvelle Revue Théologique" 106 (1984), p. 59-71; A. Feuillet, Il prologo del quarto vangelo, Assisi 1971; J.A. Martinez Camino, “A przez Niego wszystko się stało”. Stworzenie w Chrystusie, "Communio" 22 (2002), nr 2, p. 14-28; Cz. Bartnik, Dogmatyka ..., op. cit., vol. 1, p. 288; Z. J. Kijas, Początki świata i człowieka, Kraków 2004, p. 100-103.

3 È bene avvertire che la struttura proposta non è da tutti accettata: cf. i commentari. 
v. 3 sottolinea l'universalità della mediazione creatrice (,tutto... nulla”), così il v. 17 rileva l'universalità salvifica, perché ,grazia e verità" (che corrispondono all'ebraico hesed - 'emeth) compendiano tutti i beni salvifici, e sono donati in virtù di una mediazione, quella di Gesù Cristo, che supera largamente la mediazione di Mosè. Già il v. 16 sottolineava, del resto, la sovrabbondanza e l'universalità della grazia ricevuta in Cristo: „dalla sua pienezza $(\pi \lambda \eta \dot{\rho} \omega \mu \alpha)$ tutti abbiamo ricevuto grazia su grazia”. Se quindi il prologo riprende il tema già visto dell'unità di creazione e salvezza, aggiunge come elemento nuovo l'esplicitazione dell'incarnazione del creatore - salvatore, che negli altri testi era più presupposta che affermata.

Si deve poi notare che l'orientamento giovanneo dominante, riguardo al tema della creazione, è di ordine antropologico. Infatti, il prologo introduce il tema caratteristicamente giovanneo della „vita” (v. 4) luce degli uomini" (cf. Sap 7,10.26). C'è una trasposizione antropologica del tema luce - tenebre presente in Gn $1^{5}$. Il Logos è colui che dispensa la vita in pienezza, come suggerisce con ancor più evidenza la punteggiatura da alcuni proposta per i versetti $3 \mathrm{~s}$, che porterebbe a leggere: „Tutto è stato fatto per mezzo di lui, e senza di lui niente è stato fatto. Tutto ciò che esiste in lui era vita, e la vita era la luce degli uomini [...]”, espressione che potrebbe richiamare Col 1,17: „Tutte le cose sussistono in lui". Dunque la vitalità del Logos permette non solo il venire all'esistenza delle cose, ma anche la loro sussistenza e vitalità. La medesima vita, resasi personalmente presente in Gesù (cf. 14,6), è pure il dono escatologico, in cui si rendono presenti la grazia e la verità, è il frutto promesso a quanti credono nel nome di Gesù (cf. 20,30s).

Rileviamo infine che il Logos incarnato, creatore e salvatore, è anche il rivelatore per eccellenza di Dio, è colui nel quale il Dio invisibile e inaccessibile viene finalmente „spiegato” (Gv 1,18; cf. 6,46; 10,15; 14,9). Così Giovanni, come Eb 1,1-3, riassume nel Logos tutte le caratteristiche della Parola secondo Gn 1. Si tratta di una parola che crea e, creando, rivela l'origine del creato (cf. Sap 13,1ss; Rm 1,19ss.).

In definitiva, Giovanni esprime con un linguaggio suo proprio tematiche assai simili a quelle paoline (ciò vedremo più avanti). Il Logos è all'inizio dell'essere delle cose, le mantiene in esistenza con una continua comunicazione di vita. Creazione e salvezza sono strettamente collegate in quell'incontro unico di tempo ed eternità che è l'incarnazione ${ }^{6}$. Finalmente si può notare che anche la causalità finale di Cri-

4 I termini relativi alla ,vita” ricorrono in Giovanni con una frequenza senza paragoni: „vita” 37 volte, per esempio (Mt 7; Mc 4; Lc 5); „,vivere”, 16 volte (Mt 6; Mc 2; Lc 10), „vivificare”, 32 volte (non c'è nei Sinottici); ,generare”, 17 volte (Mt 4; Mc 1; Lc 6).

5 Anche il termine kosmos indica prevalentemente, in Gv, il mondo umano, l'ambito esistenziale degli uomini; fanno eccezione 11,$9 ; 17,5.24 ; 21,25$.

6 Cf. Z.J. Kijas, Poczatki świata..., op. cit., p. 103: „Podobnie jak w pismach Starego Testamentu, w których pierwszorzędnym celem opowiadania o stworzeniu nie jest ukazanie fizycznych początków świata, ale podkreślenie wiary w Bożą wszechmoc, tak samo teologia stworzenia pism Nowego Testamentu ukazuje fundamentalne znaczenie Jezusa Chrystusa w dziejach człowieka: związanie zbawienia ze stworzeniem”. 
sto è affermata, se non nel vangelo, per lo meno in un altro passo che dipende dalla teologia giovannea, dove Cristo è presentato come ,primo e ultimo, alfa e omega”, „principio e fine di tutte le cose”(Ap 1,17 e 22,13) ${ }^{\text {. }}$.

\section{La cristologia di 1 Cor 8,6}

Nella teologia paolina ${ }^{8}$ si ritrovano anzitutto le tematiche fondamentali dell'Antico Testamento sulla creazione: la convinzione che "tutto viene da Dio" (1 Cor 11,12) e tutto appartiene a Lui (1 Cor 10,26); il tema della creazione mediante la parola (2 Cor 4,6; $\mathrm{Rm} \mathrm{4,17);} \mathrm{il} \mathrm{primato} \mathrm{della} \mathrm{soteriologia} \mathrm{rispetto} \mathrm{alla} \mathrm{cosmologia,} \mathrm{e} \mathrm{quindi}$ anche il legame tra protologia ed escatologia (il Creatore è colui che dà la vita ai morti). Parimenti si trova il tema della cura provvidente e continua di Dio verso la sua creazione ( $\mathrm{Rm} 11,36$; 1 Tim 6,13), in termini che possono far pensare al linguaggio stoico, ma che in realtà presuppongono una visione di Dio personale, paterno e trascendente, ben diversa dal panteismo stoico.

Paolo riprende la dottrina dell'analogia (cf. libro della Sapienza 13), circa la possibilità di arrivare a conoscere, "dalla creazione del mondo in poi", l'esistenza

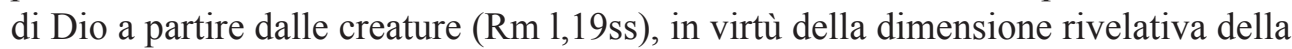
creazione. Bisogna aggiungere, però, che la creazione nella condizione attuale porta i segni del peccato, "è stata sottomessa alla caducità", è "schiava della corruzione" (Rm 8,20-21), quindi solo imperfettamente riflette lo splendore divino. È necessario pertanto che essa giunga ad essere "nuova creazione". Qui Paolo inserisce la prospettiva cristologica. Si può affermare che la visione propriamente paolina della cre-

7 G. Barbaglio riepiloga il testo analizzato in modo seguente: "il prologo del IV vangelo connota il soggetto come Parola di Dio. I riferimenti veterotestamentari alla parola mediante la quale Jahve creò il cielo e la terra (cf. Gn 1 e soprattutto Sal 33,6) e alla sapienza mediatrice della creazione si mostrano altamente attendibili, senza dimenticare un possibile influsso linguistico della cultura greca. Non si deve però disattendere l'originalità del passo evangelico che ha di mira la persona storica di Gesù di Nazaret. È lui la Parola creatrice; tutto è stato creato mediante lui. E si tratta della stessa Parola che è fonte di vita e salvezza per tutti gli uomini: 〈In lui era la vita e la vita era la luce degli uomini〉 (Gv 1,4$)$, che si è fatta presente nella storia sotto forma umana e mortale: «E la parola si fece carne e venne ad abitare in mezzo a noi $(\mathrm{Gv} 1,14)$, che gli apostoli hanno sperimentato sul piano sensibile: «Ciò che era fin da principio, ciò che noi abbiamo udito [...]»”. G. Barbaglio, Creazione, op. cit., p. 210.

8 Paolo elabora una sua riflessione cristologica che, più che un vero e proprio sviluppo, contiene tre livelli successivi di approfondimento. La sua cristologia è inoltre in funzione eminentemente soteriologica. Il primo livello, arcaico (1-2 Ts e seconda parte della 1 Cor) è incentrato sulla parusia e sulla risurrezione di Gesù, come primizia della nostra. Il secondo livello (Rm, Gal, 1-2 Cor) ha il suo centro d'interesse nell'efficacia attuale della risurrezione e della morte di Cristo, come nuovo principio religioso. Il terzo livello (Ef, Fil. Col) offre una visione cristologica più completa, rielaborando in modo nuovo i risultati dei due livelli precedenti. Cf. A. Amato, Gesù il Signore. Saggio di Cristologia, Bologna $1997^{4}$, p. 133-134. 
azione è caratterizzata dal fatto che tutto è stato creato per mezzo di Cristo e in vista di lui. L'affermazione è contenuta in 1 Cor 8,6 e in modo particolarmente accentuato nell'inno di Col 1,15-20. Per entrambi i passi si pensa comunque a un'origine prepaolina. Il ruolo cosmico di Cristo non sarebbe quindi originalmente paolino, ma avrebbe radici più antiche nella fede delle prime comunità cristiane.

In 1 Cor 8,6 troviamo per la prima volta l'indicazione di Cristo accostato al Padre nel ruolo di Creatore: "Per noi c'è un solo Dio, il Padre, dal quale tutto proviene e noi siamo per lui; e un solo Signore Gesù Cristo, in virtù del quale esistono tutte le cose e noi esistiamo per lui"'. Si presenta evidentemente come una formula di fede bipolare, cioè costruita attorno al Padre e a Cristo. In primo piano sta il "noi" della comunità cristiana che professa la sua fede monoteistica e cristologica ${ }^{10}$.

La predicazione cristiana rivolta ai pagani si qualificava proprio per questi due poli, teologico e cristologico, come attesta Paolo (1Ts 1,9-10). Al nostro scopo ci interessa di più la duplice specificazione dell'unico Dio e dell'unico Signore. Si rileva in proposito una perfetta corrispondenza delle formule: tutto è da Dio - tutto è mediante Cristo; noi siamo per Dio - noi siamo mediante Cristo. Da una parte Dio e Gesù, dall'altra l'universo e la comunità cristiana. Il collegamento è dato da un triplice rapporto: di origine, di mediazione e di finalità.

Come nel passo di Rm 11,36, ci si riferisce qui a "tutta la realtà". Però mentre là tutto era riferito unicamente al Padre, qui tutta la realtà creata è vista come opera del Padre e del Figlio nello stesso tempo. Si deve osservare, però, l'uso differenziato delle preposizioni, che invita a considerare la causalità del Padre e di Cristo in modi distinti. Il Padre è principio e fine, il Cristo è mediatore della creazione, allo stesso modo in cui lo è della salvezza. Circa l'origine, il significato e la portata di questa mediazione, noi vediamo qui di seguito la presentazione di Col 1,15-20.

9 Al nostro scopo comunque interessa di più la duplice specificazione dell'unico Dio e dell'unico Signore. Si rileva in proposito una perfetta corrispondenza delle formule: tutto è da Dio/tutto è mediante Cristo; noi siamo per Dio/noi siamo mediante Cristo. Da una parte Dio e Gesù, dall'altra l'universo e la comunità cristiana. Il collegamento è dato da un triplice rapporto: di origine, di mediazione e di finalità. Il Padre è il principio creatore di tutte le cose e il fine ultimo a cui la comunità cristiana è ordinata. Gesù invece tanto nell'azione creatrice che in quella storico-salvifica gioca il ruolo di mediatore. Il testo non spiega né il come né il perché. Ci dobbiamo accontentare dell'affermazione: Gesù ha avuto a che fare anche con l'origine dell'universo. L'unico punto di riferimento è costituito dall'analoga partecipazione all'opera salvifica. Si può ragionevolmente supporre che i cristiani partendo da questo dato di fede abbiano intuito come Gesù non poteva essere assente dall'opera creatrice.

10 Per le discussioni sollevate dal testo sul suo significato puramente cosmologico o anche soteriologico cf. A. Feuillet, La profession de foi monothéiste de 1 Cor 8, 4-6, in Le Christ Sapesse de Dieu, Parigi 1966, p. 59-85. La formulazione unisce così in stretta coesione un elemento della tradizione veterotestamentaria e un motivo propriamente cristiano. Non è difficile supporre che si tratti di una professione di fede di cristiani venuti dal paganesimo, il cui cammino di adesione al Signore Gesù doveva passare attraverso il riconoscimento dell'unico Dio. 


\section{La cristologia cosmica di Col 1,15-20}

L'inno contenuto nella lettera ai Colossesi $(1,15-20)^{11}$ è un testo insieme di notevole complessità e di grande importanza, anche per l'enorme influsso che ha esercitato sugli sviluppi della cristologia ${ }^{12}$. Qualcuno ha voluto vedere in quest'inno un tema ellenistico (miti gnostici di redenzione). Sembra più corretto intenderlo come una risposta a quegli ellenisti che si ritenevano sotto il dominio di potenze malvagie che dimoravano nelle regioni superiori dell'universo. Essi non hanno motivo di timore o di ritenersi minacciati: Cristo, e non le potenze celesti, domina sull'universo; Cristo, che non può essere semplicemente identificato con l'universo, in quanto la sua opera di redenzione si compie in un atto storico, sulla croce; in Gesù Cristo l'iniziativa divina si unisce strettamente alla sua azione storico-salvifica ${ }^{13}$.

L'opinione di molti esegeti è che Col 1,15-20 sia basato su un inno prepaolino. Sono stati fatti diversi tentativi di ricostruire l'inno originario che starebbe alla base della redazione attuale ${ }^{14}$. Si tratta comunque di tentativi ipotetici. Più prudentemente, sembra che si debbano intendere come glosse, dovute al redattore di Col, solo le espressioni "cioè della Chiesa" (v. 18) e "con il sangue della sua croce" (v. 20). Un altro problema riguarda l'estensione dell'inno, che alcuni ampliano facendolo iniziare dal v. 12. Teniamo qui l'opinione prevalente, che vede l'inno delimitato ai versetti $15-20$.

L'interesse dell'inno sta nel fatto che la risurrezione e glorificazione di Cristo è presentata qui con categorie di tipo cosmologico; in questo differisce da altri inni cristologici, come Fil 2,6-11 o 1 Tim 3,16, che sono invece basati su categorie stori-

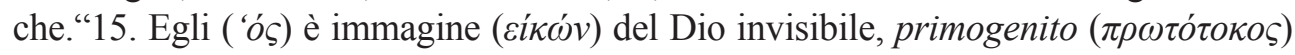

11 Per l'esegesi del testo si veda p. es.: J.L. Aurrecoechea, Los títulos cristológicos de Colosenses 1,15-16. Su origen y su significado, "Estudios Trinitarios" 8 (1974), p. 307-328; G. Segalla, La cristologia del Nuovo Testamento, Brescia 1985; Associazione Biblica Italiana, La cristologia in San Paolo. Atti della XXIII Settimana Biblica, Brescia 1976; J.N. Aletti, Saint Paul. Épître aux Colossiens, Paris 1993, p. 86-118; G. O’Collins, Cristologia. Uno studio biblico, storico e sistematico su Gesù Cristo, Brescia 1997, p. 4042; T. Otero Lazaro, Col 1,15-20 en el contexto de la carta, Roma 1999, p. 267-299; Cz. Bartnik, Dogmatyka..., vol. 1, op. cit., p. 285-286; Z.J. Kijas, Początki świata..., op. cit., p. 104-105.

12 Si veda al riguardo A. Grillmeier, Gesù il Cristo nella fede della Chiesa, vol. I: Dall'età apostolica al concilio di Calcedonia (451), Brescia 1982, p. 143-167.

13 Cf. G. von Rad, Teologia dell'Antico Testamento, vol. 1, Brescia 1974, p. 158-195.

14 A. Grillmeier, Gesù il Cristo..., op. cit., p. 137, riferisce p. es. quella di C. Burger, Schöpfung und Versöhnung. Studien z. Liturgischen Gut im Kolosser - und Epheserbrief, Neukirchen Verlag 1975. Secondo questo autore, l'inno originario suonerebbe così: "Egli è immagine / primogenito di ogni creatura / perché in lui sono state create tutte le cose. - Egli è principio / primogenito dai morti / perché in lui dimora tutta la pienezza / sia le cose della terra che quelle dei cieli"; cf. anche la diversa ricostruzione proposta da J. Ernst, sempre in Grillmeier, p. 138. 


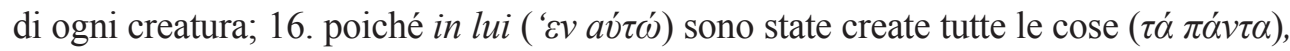
quelle nei cieli e quelle sulla terra, quelle visibili e quelle invisibili: Troni, Dominazioni, Principati e Potestà. Tutte le cose sono state create per mezzo di lui $\left(\delta l^{\prime} \alpha\right.$ av́ví) e in vista di lui ( $\varepsilon i \zeta$ ' ' $\alpha v \tau o ́ v)$. 17. Egli è prima di tutte le cose e tutte sussistono in lui (' $\varepsilon v$ av́tóv). 18. Egli è anche il capo del corpo (cioè della Chiesa); Egli ('ós) è il principio,

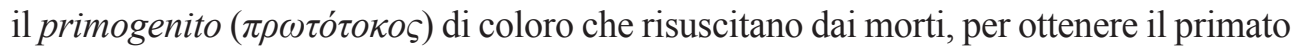

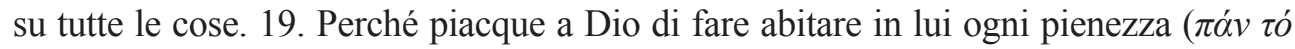
$\pi \lambda \eta \dot{\rho} \omega \mu \alpha)$, 20. e per mezzo di lui riconciliare a sé tutte le cose, rappacificando (con il sangue della sua croce, cioè) per mezzo di lui, le cose che stanno sulla terra e quelle nei cieli.

Si possono riconoscere due strofe ${ }^{15}$, che iniziano in modo simile, con l'espres-

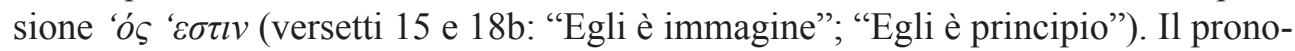
me iniziale 'ó $̧$ è caratteristico di questi inni (cf. Fil 2,5). Il v. 15 b corrisponde esattamente al testo presupposto dalla versione latina di Sir 24,9 (primogenito ante omnem creaturam), che si riferisce alla sapienza ${ }^{16}$. La prima strofa (versetti 15-18a) parla del ruolo del Figlio nei confronti della creazione. Egli precede ogni realtà creata, e tutto ciò che si trova nel cielo e sulla terra dipende da lui, in quanto è stato voluto per lui, è stato creato per suo mezzo e dipende da lui per rimanere nell'esistenza. L'altra strofa (versetti 18b-20) è d'ordine soteriologico, e considera l'opera del Figlio in ordine a una salvezza intesa (per il parallelismo con la prima strofa) come una nuova creazione.

II tema di Cristo immagine ( $\varepsilon i \kappa \omega ́ v$ ) si trova già in 2 Cor 4,4, che parla della gloria di Dio visibile sul volto di Cristo, il quale è "immagine di Dio". In altre parole, Cristo è colui che rivela pienamente il Padre. In quest'affermazione si riconosce il ruolo delle speculazioni sapienziali nel giudaismo ellenistico: la sapienza era detta "immagine di Dio". Qui è da notare, però, che il titolo non è riferito a una sapienza impersonale, ma al Cristo storico in tutta la sua realtà ${ }^{17}$, e questo in relazione al fatto che già in precedenza Cristo era stato definito come "sapienza di Dio" (cf. 1 Cor 1,24.30) ${ }^{18}$.

15 J.L. Ruiz de la Peña (seguendo P. Lamarche, Christ vivant. Essai sur la Christologie $d u N T$, Paris 1966), parla di tre strofe, e considera i vv. 16b-18 una strofa di transizione; cf. p. 64 e 66s. Sono invece due strofe anche per J.N. Aletti, Colossiens 1, 15-20. Genre et exégèse du texte. Fonction de la thématique sapientielle, Roma 1981.

${ }^{16} \mathrm{Cf}$. anche, forse, la versione latina di Sir 24,6: et in omni populo et in omni gente primatum habui.

${ }^{17}$ Si discute su chi sia propriamente il soggetto delle affermazioni dell'inno, se il Cristo preesistente o il Cristo incarnato, o eventualmente il primo per la prima strofa, il secondo per l'altra. In realtà, la distinzione pare qui fuori luogo. L'inno va tutto riferito al Cristo nella totalità del suo essere incarnato; cf. A. Grillmeier, Gesù il Cristo..., op. cit., p. 139s; J.L. Ruiz de la Peña, Christ vivant..., op. cit., p. 64s.; P. Benoit, Préexistence et incarnation, Revue Biblique 77 (1970), p. 5-29.

18 Cf. S. Cipriani, Cristo „Potenza di Dio e Sapienza di Dio” in 1 Cor 1, 24, in Associazione Biblica Italiana, La Cristologia in San Paolo. Atti della XXIII Settimana Biblica, Brescia 1976, p. 341-360; G. O’Collins, Cristologia, op. cit., p. 43-46. 
Se Cristo è detto "immagine" in base alla sua identificazione con la "sapienza", si comprende allora meglio anche il ruolo cosmico che il séguito della strofa attribuisce a Cristo. Come ha mostrato S. Lyonnet ${ }^{19}$, tutta la questione va capita alla luce del ruolo salvifico che il giudaismo attribuisce alla Torah, identificandola con la sapienza. Più ancora che il Messia, strumento di salvezza era ritenuta la Torah. Non per nulla Paolo, formatosi alla scuola dei farisei, può dire che la sua conversione consiste nell'aver sostituito Cristo alla Legge proprio in quanto mezzo di giustificazione e salvezza (cf. Fil 3,7-9). Per questo Paolo può attribuire a Cristo tutto ciò che veniva attribuito alla Legge. E se la Legge era considerata non solo strumento di giustificazione e salvezza, ma anche mediatrice di creazione per l'universo, Paolo poteva attribuire a Cristo anche questo ruolo cosmico.

Come ciò passi attraverso il riferimento alla sapienza, si può vedere in base a 1 Cor 1,30, dove a Cristo viene attribuito il ruolo determinante nella mediazione salvifica, proprio attraverso l'identificazione con la Sapienza che, a sua volta, era identificata nel giudaismo con la Torah. Il testo di 1 Cor 1,30 va inteso così: "[...] il quale (Cristo) è divenuto per noi sapienza, cioè giustizia, santificazione e redenzione". In altri termini, Cristo è sapienza (= Torah) per noi, in quanto è il vero mediatore della salvezza di cui Dio è unico autore, e che Paolo poi descrive nelle tre fasi di giustificazione, santificazione e redenzione (escatologica).

L'assimilazione tra sapienza e Torah spiega poi la funzione cosmica attribuita a quest'ultima e che Paolo ${ }^{20}$, conseguentemente, applica a Cristo. I testi sapienziali che facevano riferimento al ruolo creatore della sapienza (soprattutto $\operatorname{Pr} 8$ e Sir 24) erano stati riferiti nel giudaismo alla Torah creatrice. Si tratta di una concezione che dipende, peraltro, dal tema della creazione mediante la parola ${ }^{21}$, e dall'identificazione parola - sapienza.

Si deve porre l'accento che Paolo riferisce a Cristo il ruolo di mediazione che il giudaismo attribuiva alla Torah (identificata alla sapienza), sia sul piano salvifico, sia in ordine al tema della creazione. Perciò il Cristo - immagine (cioè sapienza) di cui parla Col 1,15 assume un ruolo determinante in riferimento alla creazione. In questo senso egli è detto "primogenito" di tutta la creazione. Il termine non indica tanto una precedenza cronologica, ma un primato di valore: „Cristo

19 S. Lyonnet, Ruolo cosmico di Cristo in Col. 1, 15ss. alla luce del ruolo cosmico della Torà nel giudaismo, in: AA.VV., La Cristologia in San Paolo. Atti della XXIII Settimana Biblica, Brescia 1976, p. 57-79; si veda anche R. Penna, Infrazione e ripresa del rapporto Legge-sapienza in Paolo, in: AA.VV., Sapienza e Torah. Atti della XXIX Settimana Bibli$c a$, Bologna 1987, p. 126-151. Penna non considera autenticamente paolina la lettera ai Colossesi. Si vedano comunque le riflessioni su 1 Cor 8,6, p. 140-142.

${ }^{20}$ La questione dell'autore della lettera ai Colossesi non è risolta. In ogni caso, il ruolo cosmico di Cristo è presente, come si è visto, anche in lettere sicuramente paoline: oltre 1 Cor 8,6, cf. anche Rm 8,19-22.

${ }^{21}$ Uno dei modi usati dal giudaismo per nominare Dio è: "Colui che disse e il mondo fu". 
è il primo, non in una serie omogenea, ma per un titolo di supremazia"22. Si deve dire, pertanto, che Cristo, nella sua unità umano-divina, è „principio” della creazione, e dunque questa non è pensabile senza incarnazione. Non è concepibile una creazione che non sia fin dall'inizio posta sotto il segno dell'incarnazione.

Tenendo presente tutto ciò, quanto segue crea minori difficoltà di comprensione. Si capisce così perché ,in lui sono state create tutte le cose" (v. 16a), in quanto Cristo è concepito (ripensando alla sapienza dell'Antico Testamento ${ }^{23}$ ) come il paradigma, il „modello" concreto e personale di tutte le cose; al tempo stesso, l'espressione „in lui" allude a quell "essere in Cristo" che esprime, in Paolo, la comunione vitale con Cristo quale condizione di salvezza alla quale l'uomo è chiamato e che, mediante l'esistenza dell'uomo come „nuova creatura”, si estende a tutte le cose facendole diventare ,nuova creazione”.

L'inno elenca poi in funzione polemica quei diversi poteri cosmici (troni, dominazioni, ecc), che altrove chiama „elementi di questo mondo" (Col 2,8.15.2022), e da cui l'uomo si sente minacciato. L'esistenza di queste realtà cosmiche non è discussa, ma in ogni caso relativizzata, dal momento che tutte le cose, visibili e invisibili, sono comunque create in Cristo e dunque sono a lui sottoposte.

Il tema viene ulteriormente precisato dall'ultima parte del v. 16, dove si afferma che tutte le cose sono create „per mezzo" e „in vista” di Cristo. L'interesse di queste parole sta nel fatto che il riferimento finale, che in 1 Cor $8,6^{24}$ era riservato al Padre, qui viene riferito a Cristo (sís $\alpha v \tau o ́ v)$ cioè al Figlio incarnato. Il mondo acquista in lui non solo unità d'origine, ma anche di destino. Fin dagli inizi il disegno di Dio si configura come il progetto di realizzare quest'unità del cosmo in Cristo. Protologia ed escatologia si saldano dunque in Cristo. Ancora una volta si vede la prospettiva soteriologica delle affermazioni bibliche relative alla creazione. Cristo è alla fine della storia di salvezza come salvatore, perché è anche all'inizio come Creatore. Resta da notare che tutto questo tema cristocentrico si completa poi teocentricamente, almeno nell'insieme della teologia paolina. Infatti, non viene riferita a Cristo l'origine ultima di tutto il dinamismo di creazione - salvezza, che rimane sempre „dal (ćk) Padre”.

D'altro lato, è pure chiaro che l'orizzonte ultimo di questo dinamismo è Dio, il Padre, che in Cristo ha voluto riconciliare a sé tutte le cose ( $\tau \dot{\alpha} \pi \dot{\alpha} v \tau \alpha$ v. 20)

22 J.L. Ruiz de la Peña, p. 64.

${ }^{23}$ Cf. Pr 8,22-23: "Il Signore mi ha creato all'inizio del suo operare, prima delle sue opere più antiche. Dall'eternità sono stata costituita, dall'inizio, prima dei primordi della terra"; Sap 9,1-2.4: "Dio dei padri e Signore di misericordia, che con la tua parola hai fatto l'universo e con la tua sapienza hai formato uomo, perché domini sulle creature fatte da te [...]. Dammi la sapienza che siede accanto ai tuoi troni, e non mi escludere dal numero dei tuoi servi".

${ }^{24}$ Cf. Rm 11,36: "Poiché tutte le cose provengono da Lui, esistono in grazia di Lui, tendono a Lui. A Lui gloria per i secoli". 
fino ad essere „tutto in tutti” ${ }^{25}$. Il versetto 17 completa il discorso affermando il primato (anche temporale) di Cristo, la sua signoria sull'intera creazione, la quale trova in lui la sua sussistenza, nel senso che è Cristo il fondamento continuo della permanenza di tutte le cose nell'essere, il principio della coesione, dell'unità e armonia di tutto. Per questo motivo, Cristo è indicato pure come il „,capo del corpo” (v. 18a). Nel termine „corpo” dobbiamo presumibilmente vedere un influsso del vocabolario stoico, che indica con questo termine $\left(\sigma \omega^{\prime} \mu \alpha\right)$ l'universo nella sua immanente strutturazione. L'espressione „cioè della Chiesa” suona evidentemente come un correttivo, spostando l'attenzione sul corpo della comunità ecclesiale ${ }^{26} \mathrm{di}$ cui Cristo è il capo. Ciò non diminuisce la dimensione cosmica di questo essere „capo" di Cristo, ma esprime il modo ecclesiologico in cui questa prerogativa si esercita.

Al versetto $18 \mathrm{~b}$ il discorso riprende di nuovo col pronome: „Egli è...”. A Cristo

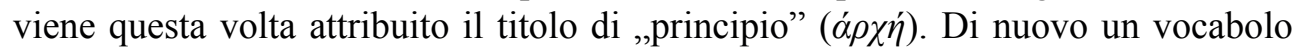
probabilmente preso dal linguaggio stoico, dove spesso equivale a „testa”, e che ancora una volta viene corretto mediante l'aggiunta di ,primogenito di coloro che risuscitano dai morti”. Rispetto al v. 15b (,primogenito di ogni creatura”), qui il discorso si sposta decisamente sull'asse soteriologico. Si confronti anche Rm 8, 29 (,primogenito tra molti fratelli"), notando però che in Colossesi la primogenitura è allargata al rapporto con ,tutte le creature”. Si vede dunque che la realtà è doppiamente cristologica. In ragione della creazione (protologia) e in ragione della risurrezione (escatologia), che diventa pertanto una „nuova creazione” il cui principio è sempre Cristo.

Il termine $\pi \lambda \dot{\eta} \rho \omega \mu \alpha$ (,pienezza”, v. 19), anch'esso appartenente al vocabolario stoico, deve essere interpretato alla luce di Col 2,9s: „In Cristo abita corporalmente tutta la pienezza della divinità, e voi avete in lui parte alla sua pienezza, di lui cioè che è il capo d'ogni Principato e d'ogni Potestà". Si tratta di una pienezza comunicativa, che è stata data a Cristo perché egli ne renda partecipi le creature. Si tratta della pienezza che si ottiene in virtù della riconciliazione di tutte le cose (v. 20), che è mediata da Cristo e orientata a lui ${ }^{27}$, e ultimamente al Padre, come già lo era la crea-

251 Cor 15,28: "E quando tutto gli sarà stato sottomesso, anche egli, il Figlio, farà atto di sottomissione a Colui che gli ha sottomesso ogni cosa, affinché Dio sia tutto in tutti".

261 Cor 12,12: "Come il corpo, pur essendo uno, ha molte membra, e tutte le membra, pur essendo molte, sono un corpo solo, così anche il Cristo"; Ef 1,22-23: "Ha posto tutto sotto i suoi piedi e lo ha costituito, al di sopra di tutto, capo della chiesa, che è il suo corpo, la pienezza di lui che tutto, sotto ogni aspetto, riempie".

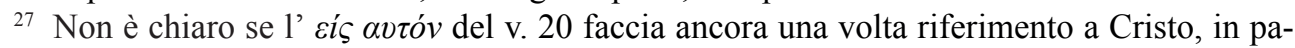

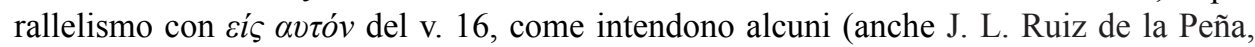
p. 69), o se invece sia da riferire a Dio. In ogni caso, l'orientamento teocentrico del discorso è sempre presente; cf. 2 Cor 5,19, dove si parla della riconciliazione del mondo con Dio

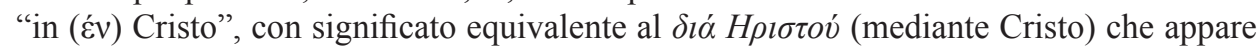
subito prima. 
zione. Potremmo schematizzare il movimento del discorso, integrandolo lievemente

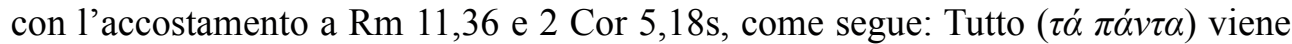
da $(\dot{\varepsilon} \kappa)$ Dio (il Padre) $(\mathrm{Rm} 11,36)$ mediante ( $\delta i \alpha ́)$ Cristo; tutto ( $\tau \dot{\alpha} \pi \dot{\alpha} v \tau \alpha)$ sussiste in Cristo e in vista ( $\varepsilon i \varsigma)$ di Cristo perché tutto ( $\tau \dot{\alpha} \pi \dot{\alpha} v \tau \alpha)$ sia riconciliato mediante $(\delta i \alpha ́)$ Cristo [in ( $\dot{v} v)$ Cristo: 2 Cor 5,19] e in vista ( $\varepsilon i \varsigma)$ di Cristo [con Dio (il Padre): 2 Cor 5,19].

Come si vede, l'inno esaminato insiste molto sull'unità di creazione e salvezza in Cristo. L'insistenza è motivata probabilmente da un intento antignostico. Si tratterebbe di contrastare il dualismo gnostico, che separa nettamente e contrappone la creazione (vista negativamente) e la redenzione ${ }^{28}$. A questa visione, l'inno contrappone una grande concezione unitaria, quella di una realtà integrata e ricapitolata in un principio personale che le conferisce dall'inizio alla fine significato, consistenza e pienezza, che „crea per salvare e salva quanto ha creato" 29 .

\section{La cristologia di Eb 1,1-3}

L'esordio della lettera agli Ebrei incentra il suo interesse sull'avvenimento della rivelazione divina definitiva in Gesù Cristo, contrapposta alla fase preparatoria

${ }_{28}$ Al dualismo cosmico della gnosi corrisponde un dualismo teologico (il Demiurgo autore della creazione è un dio diverso, e inferiore, rispetto al Dio Padre di Gesù), antropologico (spiritualità e materialità dell'uomo si oppongono), scritturistico (opposizione tra Antico Testamento e Nuovo Testamento), ecc.

29 Vogliamo qui anche menzionare l'altro testo paolino. Si tratta dell'inno di ringraziamento di Ef 1,3-14, che esplicita ancora più chiaramente di Colossesi la vocazione cristologica di tutta la creazione. Il testo è molto complesso - e andrà ripreso anche in altri contesti, per esempio per la problematica della predestinazione. È chiaro in ogni modo che qui s'intende parlare del disegno eterno di Dio sulla creazione. Questo disegno, che ,precede” la creazione stessa (versetti 4. 9. 11) ha come suo contenuto il „ricapitolare in Cristo tutte le cose” (v. 10), far confluire in Cristo il destino di tutto il creato (cf. versetti 22s.). Come in Colossesi, si allude qui anche ad una realizzazione ecclesiologica di questo progetto divino. L'intero universo è beneficiario della pienezza di Cristo, la quale, però, è presente anzitutto nella Chiesa e si diffonde per suo mezzo in tutto il creato, nella misura in cui la Chiesa diventa in modo sempre più pieno il Corpo di Cristo. L'espressione più ricorrente nel testo che fa proprio il centro di tutto, è ,,in lui” (versetti 3s.,6s.,9,10,11,12,13). Si tratta dell'espressione che assume poi diverse sfumature, convergenti nell'indicare Cristo come il mediatore universale ed esclusivo di tutta l'attività divina ad extra, sia nel suo inizio (protologia) che nel suo compimento storico e nel suo compimento (escatologia). In questo senso al v. 9 ci si riferisce al „mistero" per eccellenza, che è sempre Cristo, in quanto è il destino irrevocabile di tutto. Cf. F. F. Bruce, The Epistles to the Colossians, to Philemon, and to the Ephesians, Grand Rapids 1984; J. Gnilka, Der Epheserbrief, Freiburg 1982; C.L. Mitton, Ephesians, Greenwood 1976; H. Schlier, La lettera agli Efesini, Brescia 19732; R. Schnackenburg, Der Brief an die Epheser, Neukirchen 1982; Cz. Bartnik, Dogmatyka..., vol. 1, op. cit., p. 286. 
e imperfetta dell'Antico Testamento. Circa il testo di Eb 1,1-3, assai vicino a Col 1,15-20, si deve sottolineare che la differenza fondamentale tra i due testi è data dal fatto che Eb incentra tutto sul tema della "parola" che non solo parla e rivela, ma anche "sostiene tutto". Così nel Figlio glorificato si concentrano tutte le caratteristiche della parola di Dio nell'Antico Testamento: creazione, rivelazione, sostegno, salvezza.

Possiamo far risaltare in sintesi alcuni aspetti importanti per il nostro tema. Prima di tutto è certo che il soggetto in questione è Gesù di Nazaret morto e risuscitato. Appellarsi a una distinzione tra il Gesù storico e il Verbo divino, attribuendo a quest'ultimo quanto l'inno afferma della funzione mediatrice di Cristo nella creazione del mondo, non rende giustizia al testo biblico. Il canto di lode vuole esaltare il primato cosmico (〈primogenito d'ogni creatura)) e soteriologico (〈primogenito tra i morti〉) di Gesù visto nella sua unità storica. La triplice particella poi con cui viene espresso il suo rapporto con il mondo, in, mediante, per, potrebbe indirizzare a vedervi l'affermazione di un triplice nesso di causalità esemplare, efficiente, finale, e far pensare a Cristo come archetipo, principio operativo e traguardo finalistico del creato.

Tuttavia il carattere innico del passo fa propendere per un'interpretazione meno precisa. Gesù qualifica globalmente la creazione dell'universo. Dio resta il principio supremo di tutte le cose, stante il senso teologico del passivo del verbo <è stato creato', che sottintende l'azione divina del Padre. Si tratta di un mondo creato nella prospettiva di Cristo. Al di fuori di lui nessuna cosa è pensabile, niente potrebbe esistere. Infine si deve valutare il parallelismo stabilito espressamente dall'inno tra la mediazione cosmica di Gesù e la sua mediazione nell'opera salvifica.

Possiamo concludere, seguendo le riflessioni di G. Barbaglio, che nella prima lettera ai Corinzi la funzione mediatrice nella creazione era attribuita al Signore, nel prologo giovanneo alla Parola, invece nel testo analizzato al Figlio. Per mezzo suo Dio ha creato il mondo e si è rivelato pienamente. Ne risulta che protologia ed escatologia si corrispondono e perciò la fine richiama l'inizio. Si tratta di un'unica storia, specificata dall'iniziativa di grazia di Dio e della mediazione del Figlio. Se questi è la parola ultima non può non aver rapporto anche con la prima e con tutte le parole intermedie. Egli è l'erede in senso totale, cioè realizzando tutte le promesse le finalizza a sé e così le caratterizza ${ }^{30}$.

\section{Nuova creazione in Cristo}

Si deve accertare che la cristologia della creazione permise alle prime comunità cristiane di rendere comprensibile nel contesto ebraico ed ellenistico la propria professione di fede che in Gesù Cristo tutta l'umanità è liberata dalle catene della morte e del peccato. Gli scritti neotestamentari si collocano perciò nell'orizzonte salvifico universale degli scritti veterotestamentari e confessano Gesù Cristo come il Figlio

$\overline{30}$ G. Barbaglio, Creazione, in Nuovo Dizionario di Teologia, Cinisello Balsamo 1994, p. 211. 
di Dio, attraverso il quale tutto è stato creato e che, in qualità d'erede dell'universo, tutto raduna ${ }^{31}$.

Per quanto sia vero che l'orizzonte cosmico non costituisce il punto di vista dominante e l'asserto primario della speranza cristiana escatologica, è pur vero che mai nella Bibbia la salvezza dell'uomo è pensata senza mondo. Sarebbe in contrasto con la visione della Scrittura, avallata dal pretesto di voler decosmologizzare le forme espressive della speranza, giungere ad una radicale demitizzazione delle sue dimensioni cosmiche. Come la prima creazione dell'uomo è infatti essenzialmente legata alla creazione del mondo che non è un semplice scenario dell'opera creativa di Dio, ma una sua dimensione intrinseca a cui l'uomo è congiunto proprio come "corpo" ed "immagine di Dio", così la parusia in cui la prima e la seconda creazione trovano il loro compimento escatologico, ha una sua dimensione essenziale proprio nella realtà cosmica.

La nuova creazione non è veduta nel piano di Dio come una "sostituzione" o semplice "riparazione" della prima, sfigurata dal peccato. La nuova creazione è piuttosto il "compimento" del grande progetto cosmogenetico di Dio che a partire dal primo momento creativo si protrae nel corso del tempo fino alla conclusione parusiaca. Tra primo momento creativo e sua conclusione parusiaca non c'è unicamente diastasis e frattura, ma continuità e progresso ${ }^{32}$. G. Sauter rileva: "La creazione diviene $[. .$.$] tema prospettico del tempo e non si lascia ridurre assolutamente ad un$ ampliamento retrospettivo della fede nella salvezza [...]; la promessa rivolta alla creazione elimina ogni sospetto che l'escatologia altro non sarebbe se non una forma di scetticismo larvato, una riserva che priva di ogni suo valore la salvezza presente, anzi, la presenza stessa di Dio, in quanto rimanda continuamente al futuro. Il sì del creatore è più vigoroso, più importante, più dinamizzante del non-ancora del mondo"33.

L'anonimo profeta dell'esilio aveva sollecitato gli esuli a distogliere lo sguardo nostalgico dal passato e a fissarlo sul futuro, che Dio stesso avrebbe creato: "Non ricordate più le cose passate, non pensate più alle cose antiche! Ecco faccio una cosa nuova: proprio ora germoglia, non ve ne accorgete?" (Is 43,18-19). Si trattava dell'annuncio di una nuova creazione. In ambiente apocalittico poi i visionari avevano testimoniato la nascita di un nuovo mondo. Li aveva preceduti il Tritoisaia: "Ecco infatti io creo nuovo cielo e nuova terra; non si ricorderà più il passato, non verrà più in mente" (Is 65,15).

Nel Nuovo Testamento è Paolo che più si trova in sintonia con questo flusso di speranze e attese, di cui afferma il compimento e la realizzazione in Gesù Cristo ${ }^{34}$.

31 AA.VV., Nuovo corso di dogmatica. Dottrina della creazione, p. 180.

32 Cf. M. Bordoni-N. Ciola, Gesù nostra speranza. Saggio di escatologia, Bologna 1998, p. 246-247.

33 G. Sauter, Zukunft und Verheißung. Das Problem der Zukunft in der gegenwärtigen theologischen und philosophischen Diskussion, Zürich 1965, p. 175; J. Moltmann, Futuro della creazione, Brescia 1980.

${ }^{34}$ Cf. B. Rey, Créés dans le Christ Jésus. La creation nouvelle selon st Paul, Parigi 1966. 
La creazione escatologica è una cosa presente: "Quindi se uno è in Cristo, è una nuova creatura; le cose vecchie sono passate, ecco ne sono nate di nuove" (2 Cor 5,17). Cristo è l'uomo nuovo creato in conformità al progetto divino. Aderendo a lui nella fede e nella carità il credente diventa membro vivo di un'umanità nuova.

Resta sempre vero che la novità cristiana non è un possesso definitivo e perfetto. $\mathrm{Al}$ contrario essa sussiste nella precarietà, parzialità e imperfezione. Il nuovo mondo si è fatto presente nella nostra storia, ma il vecchio mondo continua. Il creato è come una partoriente che geme ora nei dolori del parto, in attesa della sua piena redenzione. Nella lettera ai Romani si legge: "Il creato attende con impazienza la rivelazione dei figli di Dio $[. .$.$] e nutre la speranza d'essere liberato dalla schiavitù della corru-$ zione, per entrare nella libertà della gloria dei figli di Dio. Sappiamo bene infatti che tutto il creato geme e soffre fino ad oggi nelle doglie del parto; esso non è il solo, ma anche noi, che possediamo le primizie dello Spirito, gemiamo interiormente aspettando l'adozione a figli, la redenzione del nostro corpo. Poiché nella speranza noi siamo stati salvati" ( $R \mathrm{~m} 8,19-24)$. La nuova creazione dei tempi finali si è veramente anticipata nella storia per opera di Cristo. L'esperienza cristiana attuale è caparra e pegno del compimento finale quando appariranno in tutto il loro splendore i cieli nuovi e la terra nuova ${ }^{35}$.

L'affermazione del principio di continuità tra i nuovi cieli e la nuova terra con l'opera presente dell'uomo non deve però avallare forme d'incarnazionismo terrestre che confondano il progresso e l'umanizzazione del mondo con una marcia trionfale che troverà il suo sbocco nella Gerusalemme celeste. Due cose non si devono ignorare: che esiste anzitutto una discontinuità tra progresso mondano ed avvento del regno di Dio e quindi che l'avvento dei nuovi cieli e della nuova terra, nella speranza cristiana richiama l'intervento di un "nuovo atto creatore divino" o forse meglio, l'adempimento dell'opera creatrice di Dio, per cui non c'è mondo davvero compiuto senza questo "nuovo intervento".

\section{Considerazioni conclusive}

La riflessione sull'evento di Cristo, sviluppata nelle prime comunità cristiane alla luce dell'esperienza pasquale, utilizzò idee e modi di esprimersi desunti dal mondo ebraico. In particolare le speculazioni radicate nella tradizione ellenistico-ebraica sull'importanza della sapienza (sophía) e della parola (lógos) nell'attività creatrice di Dio si dimostrarono possibili punti d'aggancio per un'interpretazione dell'evento di Cristo in chiave di teologia della creazione.

Solo pochi rimandi neotestamentari alla realtà della creazione non fanno ricorso in un contesto chiaramente cristologico, ma richiamano alla mente l'idea già sviluppata in Sap 13, secondo cui la grandezza e la gloria di Dio possono essere percepite osservando le opere della creazione (cf. Rm 1,20). Tale argomento ha il suo "luogo" nella missione cristiana primitiva verso i pagani. Secondo gli Atti degli Apostoli Pa-

35 Cf. M. Bordoni-N. Ciola, Gesù nostra..., op. cit., p. 247. 
olo cercò di indurre i suoi ascoltatori non ebrei a convertirsi al Dio vivente, "che ha fatto il cielo, la terra, il mare e tutte le cose che in essi si trovano" (At 14,15).

Esaminando nel suo insieme il messaggio biblico sulla creazione, possiamo in primo luogo affermare che tale messaggio non impone una determinata concezione fisica del mondo. L'orizzonte fondamentale del messaggio biblico è d'ordine soteriologico, come si vede con la massima chiarezza nel Nuovo Testamento, il quale opera una concentrazione cristologica delle affermazioni di fede relative alla creazione. Proprio questa concentrazione cristologica - prospettiva cristocentrica indica che il cristiano non può riflettere sulla fede nella creazione semplicemente a partire dai testi dell'Antico Testamento, perché la chiave di lettura decisiva della creazione è data per lui da Cristo. Proprio questa prospettiva permette anche di evitare una serie di malintesi inutili e fuori luogo nei rapporti tra fede e scienza e, più in generale, tra fede e cultura.

Un elemento costante da notare nella fede biblica relativa alla creazione è la sottolineatura della bontà della creazione. Il pensiero biblico esclude ogni considerazione radicalmente pessimistica o fatalistica della creazione. Naturalmente, ciò comporta come conseguenza la maggior difficoltà che la fede cristiana ha nell'affrontare il problema del male. In definitiva, una spiegazione del problema del male come quella manichea si rivela estremamente semplificante. La Scrittura non ignora certo questo problema, anzi, invita a non semplificarlo, e a vederlo nella prospettiva più ampia della salvezza e dell'escatologia. Il che non è una fuga dal problema perché, come si è visto, il discorso sulle origini è strettamente legato al discorso sulla salvezza (soteriologia) e sulla fine (escatologia). La stessa considerazione di Cristo come unico „principio e fine” di tutte le cose spinge lo sguardo in avanti, verso la promessa di una redenzione della creazione da tutte le espressioni di male che la affliggono nel presente. È insieme invito a respingere ogni dualismo, per l'origine come per la fine, e quindi a non staccare tra di loro l'ordine della „natura” e quello della „grazia e verità" - il che non significa affatto, peraltro, giungere ad una identificazione pura e semplice di questi due ordini.

L'ottimismo della fede biblica dipende in definitiva dal fatto che la creazione è posta sotto il segno di una promessa, la quale assicura il futuro della creazione e fonda la speranza di un compimento. Le diverse cosmogonie extrabibliche sono per lo più rivolte al passato, laddove il pensiero biblico guarda al passato (tutto è stato fatto per mezzo di Cristo), ma considera anche il presente (tutto sussiste in Cristo) e, soprattutto, tende al futuro, quando la creazione intera sarà associata alla gloria di quanti, in Cristo, sono chiamati ad essere figli di Dio (cf. Rm 8,19-30).

Dall'unità cristologica dell'ordine della creazione e di quello della salvezza, deriva anche una conseguenza nell' ordine della prassi. II luogo in cui riceve risposta la questione riguardante la realtà di Dio, così come quella che si riferisce alla realtà del mondo, è indicato soltanto dal nome: Gesù Cristo. In questo nome sono inclusi Dio e il mondo. In lui tutto ha la sua consistenza. D'ora in poi non si può più parlare correttamente né di Dio né del mondo senza parlare di Gesù Cristo. Si tratta, d'altra 
parte, di superare l'alternativa „o Cristo o il mondo”, che ha caratterizzato gran parte della teologia e spiritualità cristiana. Finché Cristo e il mondo vengono concepiti come due sfere che si scontrano o si escludono a vicenda, all'uomo resta soltanto questa possibilità: rinunciando all'insieme della realtà, situarsi in uno dei due ambiti: o ama Cristo senza il mondo, o ama il mondo senza Cristo. Questo concetto di sfere contraddice nel modo più profondo il concetto biblico. Non vi sono due realtà, ma una realtà soltanto, e questa è la realtà di Dio nella realtà del mondo che si è rivelato in Cristo. La realtà di Cristo comprende in sé la realtà del mondo. Il mondo non ha una realtà propria indipendente dalla rivelazione di Dio in Cristo.

Si tratta, in definitiva, di partire dalle affermazioni bibliche relative all'unità in Cristo di creazione o salvezza, alla finalizzazione della realtà e della storia in Cristo, alla reale anticipazione nell'esistenza cristiana del destino escatologico che attende l'uomo nella sua unità corporeo-spirituale, per sviluppare una teologia della storia e del progresso umano, capace di orientare in modo significativo la prassi storica, sociale, culturale, politica dei cristiani. Così che essi non debbano sentirsi eternamente lacerati fra la „fede divina” e la „passione terrestre” (Teilhard de Chardin), proprio perché Cristo ha portato a compimento in sé la riconciliazione di „tutte le cose, quelle del cielo come quelle della terra".

\section{Letteratura}

AA.VV., Nuovo corso di dogmatica. Dottrina della creazione, a cura di G. Canobbio, A. Maffeis. Brescia 1995.

Aletti J.N., Colossiens 1, 15-20. Genre et exégèse du texte. Fonction de la thématique sapientielle, Roma 1981.

Aletti J.N., Saint Paul. Épitre aux Colossiens, Paris 1993.

Amato A., Gesù il Signore. Saggio di Cristologia, Bologna $1997^{4}$.

Associazione Biblica Italiana, La cristologia in San Paolo. Atti della XXIII Settimana Biblica, Brescia 1976.

Aurrecoechea J.L., Los títulos cristológicos de Colosenses 1,15-16. Su origen y su significado, "Estudios Trinitarios" 8 (1974), p. 307-328.

Barbaglio G., Creazione, in Nuovo Dizionario di Teologia, a cura di G. Barbaglio e S. Dianich, Roma 19947, p. 200-213.

Bartnik Cz., Dogmatyka katolicka, vol. 1, Lublin 1999.

Benoit P., Préexistence et incarnation, "Revue Biblique" 77 (1970), p. 5-29.

Bordoni M.-Ciola N., Gesù nostra speranza. Saggio di escatologia, Bologna 1998.

Bruce F.F., The Epistles to the Colossians, to Philemon, and to the Ephesians, Grand Rapids 1984.

Burger C., Schöpfung und Versöhnung. Studien z. Liturgischen Gut im Kolosser und Epheserbrief, Neukirchen Verlag 1975. 
Cipriani S., Cristo „Potenza di Dio e Sapienza di Dio” in 1 Cor 1, 24, in Associazione Biblica Italiana, La Cristologia in San Paolo. Atti della XXIII Settimana Biblica, Brescia 1976, p. 341-360.

De La Potterie I., Studi di cristologia giovannea, Genova 1986.

Delmirani M., Creazione in Cristo. Aspetti fondamentali di Teologia biblica sulla Creazione, Roma 1986.

Feuillet A., Il prologo del quarto vangelo, Assisi 1971.

Feuillet A., La profession de foi monothéiste de 1 Cor 8, 4-6, in: Le Christ Sapesse de Dieu, Parigi 1966, p. 59-85.

Gnilka J., Der Epheserbrief, Freiburg 1982.

Grillmeier A., Gesù il Cristo nella fede della Chiesa, vol. 1, Dall'età apostolica al concilio di Calcedonia (451), Brescia 1982, p. 143-167.

Kijas Z.J., Początki świata i człowieka, Kraków 2004.

Lyonnet S., Ruolo cosmico di Cristo in Col. 1, 15ss. alla luce del ruolo cosmico della Torà nel giudaismo, in: AA.VV., La Cristologia in San Paolo. Atti della XXIII Settimana Biblica, Brescia 1976, p. 57-79.

Martinez Camino J.A., “A przez Niego wszystko się stato”. Stworzenie w Chrystusie, "Communio" 22 (2002), vol 2, p. 14-28.

Mateos J. - Barreto J., Dizionario teologico del Vangelo di Giovanni, Assisi 1982, p. 56-65.

Mitton C.L., Ephesians, Greenwood 1976.

Moltmann J., Futuro della creazione, Brescia 1980.

Neyrand G., Le sens des "logos" dans le prologue de Jean, "Nouvelle Revue Théologique" 106 (1984), p. 59-71.

O'Collins G., Cristologia. Uno studio biblico, storico e sistematico su Gesù Cristo, Brescia 1997.

Otero Lazaro T., Col 1, 15-20 en el contexto de la carta, Roma 1999.

Penna R., Infrazione e ripresa del rapporto Legge-sapienza in Paolo, in: AA.VV., Sapienza e Torah. Atti della XXIX Settimana Biblica, Bologna 1987, p. 126-151.

Rad von G., Teologia dell'Antico Testamento, vol. 1, Brescia 1974.

Rey B., Créés dans le Christ Jésus. La creation nouvelle selon st Paul, Parigi 1966.

Sauter G., Zukunft und Verheißung. Das Problem der Zukunft in der gegenwärtigen theologischen und philosophischen Diskussion, Zürich 1965.

Schlier H., La lettera agli Efesini. Brescia 1973².

Schnackenburg R., Der Brief an die Epheser, Neukirchen 1982.

Schnackenburg R., Il vangelo di Giovanni, Brescia 1973.

Segalla G., La cristologia del Nuovo Testamento, Brescia 1985. 


\section{Streszczenie}

Nauczanie Nowego Testamentu w zakresie teologii stworzenia koncentruje się na wydarzeniu Jezusa Chrystusa. Pisma Nowego Testamentu ukazują „Boga, który ożywia umarłych i powołuje do istnienia to, czego jeszcze nie ma" (Rz 4,17). Przesłanie Nowego Testamentu przedstawia zbawcze działanie Boga w Jezusie Chrystusie, także w kontekście teologii stworzenia. Wzajemna korelacja stworzenia i historii zbawienia jest niezbędna dla zrozumienia przesłania teologii stworzenia, które w rzeczywistości osadza się na zapowiedzi odkupienia dokonanego przez Jezusa Chrystusa. Innymi słowy, refleksja nad stworzeniem wypływa z refleksji nad zbawieniem, które jest nierozerwalnie związane z wydarzeniem Chrystusa.

Uznanie Chrystusa za jedyny ,początek i koniec” wszystkich rzeczy, kieruje nasze spojrzenie w kierunku obietnicy odkupienia stworzenia od wszelkich przejawów zła, które dotyka je obecnie. Jest jednocześnie zaproszeniem do odrzucenia wszelkiego dualizmu. Nie można zatem „odrywać” od siebie porządku „,natury” oraz „łaski i prawdy", z drugiej zaś strony, nie oznacza to wszystko prostej identyfikacji tych dwóch porządków.

Słowa klucze: Jezus Chrystus, Nowy Testament, teologia stworzenia

\section{The Christocentric Perspective of Creation in the New Testament}

\section{Summary}

New Testament teaching on the theology of creation underlines the event of Jesus Christ. The New Testament Scriptures describe "God who gives life to the dead and calls into being things that were not" (Rom 4:17). The New Testament message presents God's saving action in Jesus Christ, also in the context of the theology of creation. The inter-correlation of creation and salvation history is essential to understanding the message of creation theology, which is actually based on the announcement of the redemption accomplished by Jesus Christ. In other words, reflection on creation flows from reflection on salvation, which is inseparable from the Christ event.

Recognizing Christ as the only "beginning and end" of all things directs our gaze toward the promise to redeem creation from all dimensions of evil that affects it today. It is at the same time an invitation to reject all duality. Therefore, one cannot "separate" the order of "nature" and "grace and truth" from each other, however, this does not mean a simple identification of these two orders.

Key words: Jesus Christ, New Testament, theology of creation 